\title{
Wide Field-of-View Reflection-Mode Ptychographic Imaging Microscope with Tabletop 12.7 nm High Harmonic Illumination
}

Michael Tanksalvala ${ }^{1 \dagger}$, Christina L. Porter ${ }^{1 \dagger}$, Michael Gerrity ${ }^{1}$, Galen P. Miley ${ }^{2}$, Xiaoshi Zhang ${ }^{3}$, Charles S. Bevis ${ }^{1}$, Elisabeth R. Shanblatt ${ }^{1}$, Robert Karl Jr. ${ }^{1}$, Giulia F. Mancini ${ }^{1}$, Daniel A. Adams ${ }^{1}$, Henry C. Kapteyn ${ }^{1,3}$, Margaret M. Murnane ${ }^{1,3}$

1. JILA, University of Colorado, Boulder USA.

2. Dept. of Chemistry, Northwestern University, Evanston USA.

3. Kapteyn-Murnane Laboratories, Boulder USA.

High-resolution imaging is an invaluable tool for understanding nanoscale systems. In particular, tabletop extreme ultraviolet (EUV) coherent diffractive imaging (CDI) techniques based on high harmonic generation (HHG) can combine femtosecond (fs) pulse durations with nanometer resolution and elemental-, spin-, electronic- and magnetic-sensitivity, proving to be an ideal probe of complex nanostructured systems [1-2]. In this work, we demonstrate a novel technique for glancing-incidence, large field-of-view, reflection-mode ptychographic imaging using a tabletop $12.7 \mathrm{~nm}$ HHG source. To our knowledge, this is the first demonstration of reflection-mode imaging at $12.7 \mathrm{~nm}$ on a tabletop, as well as the first $\sim 13 \mathrm{~nm}$ reflection-mode image using any source of an extended sample of general composition (ie., not fabricated on a multilayer mirror).

In CDI, coherent illumination diffracts from a sample, and the resulting light is recorded on a camera (typically a CCD) placed far from the sample. The phase profile of this light is lost by the CCD, resulting in the well-known phase problem [3]. The complex field can be computationally recovered and back-propagated to reconstruct the phase and amplitude of the field leaving the object. Ptychography is a robust form of CDI in which multiple diffraction patterns are recorded as the illumination is scanned, with overlap between positions, across the sample [4]. The resulting data is combined to provide the phase and amplitude of both the sample and the illumination.

CDI traditionally suffers from an oversampling constraint - that is, a requirement that the diffracted intensity is Nyquist-sampled on the detector-which limits the allowed beam size. This restricts the achievable field of view per scan position, increasing the required data acquisition time and system stability to image large areas with high resolution. We show here that using skimming incidence reflection geometries allows this oversampling constraint to be significantly loosened, enabling rapid, wide field of view imaging. Furthermore, few materials are reflective to $13 \mathrm{~nm}$ and shorter wavelength light at non-skimming incidence angles $\left(>20^{\circ}\right.$ from the sample surface $)$, making it important to develop reflection-mode imaging techniques that work in skimming geometries.

In this work, we generated $12.7 \mathrm{~nm}$ illumination using an actively-stabilized, high-harmonic generation (HHG) source (KM Labs XUUS 4.0) driven by $2.3 \mathrm{~mJ}, 38 \mathrm{fs}, 3 \mathrm{kHz}$ light centered at $795 \mathrm{~nm}$ (KM Labs Wyvern). The residual infrared light was eliminated by two glancing-incidence silicon mirrors coated with $\mathrm{Ru}$, followed by two, 200nm thick $\mathrm{ZrO}_{2}$ filters. We show our imaging geometry in Fig. 1a. Two flat, harmonic-selecting mirrors direct $12.7 \mathrm{~nm}$ light into a glancing-incidence ellipsoidal mirror that focused our beam to $6.5 \mu \mathrm{m}$ (tall) $\mathrm{x} 11.5 \mu \mathrm{m}$ (wide). The beam illuminated the sample $9.5^{\circ}$ from glancing, and scattered onto a detector (Andor IKON-L) $84.4 \mathrm{~mm}$ from the sample. We recorded diffraction patterns for 122 scan positions in a Fermat spiral pattern with $1.56 \mu \mathrm{m}$ between positions.

$\dagger$ These authors contributed equally to this work. 


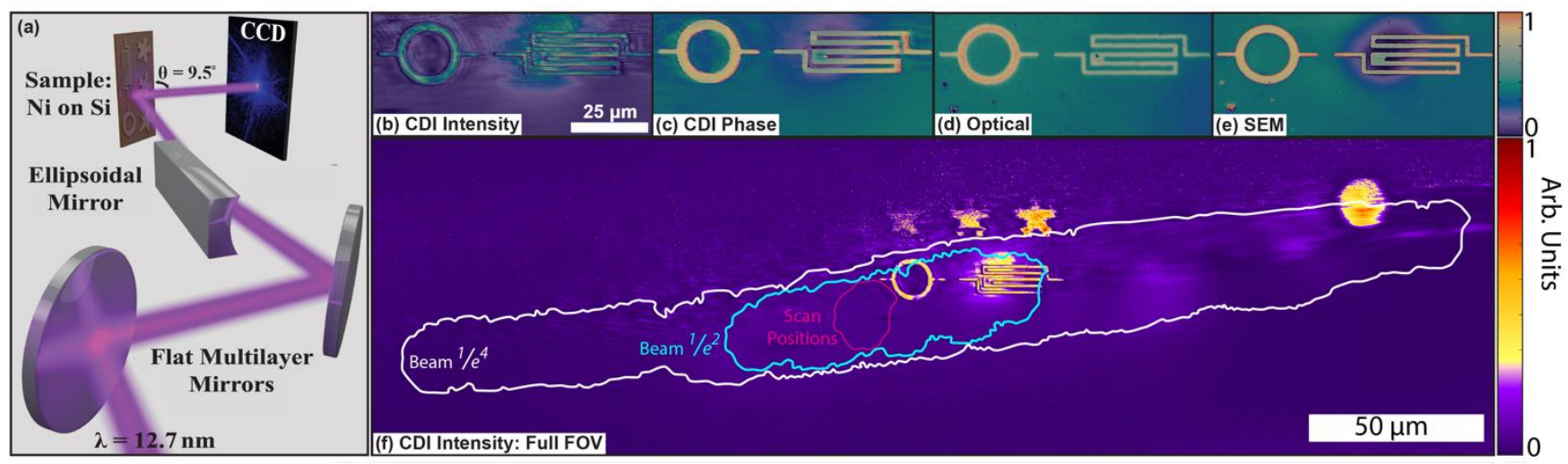

Figure 1. (a) Experimental geometry of reflection mode microscope. (b) Ptychography CDI intensity and (c) phase. (d) Optical microscope image and (e) SEM for comparison. (f) Full field of view ptychography CDI intensity image with overlaid outline of where the center of the beam was scanned (magenta), and the region over which the $1 / \mathrm{e}^{2}$ (cyan) and $1 / \mathrm{e}^{4}$ (white) diameters were scanned.

Our sample consisted of $29 \mathrm{~nm}$ tall nickel structures deposited on silicon. Because we image at a skimming incidence of $9.5^{\circ}$, the projection of our $11.5 \mu \mathrm{m}$ wide beam on the sample had a $1 / \mathrm{e}^{2}$ diameter of $70 \mu \mathrm{m}$. This beam width would not obey the traditional oversampling criterion of ptychography were it normally incident on the sample. However, at skimming incidence, conical diffraction improved the oversampling on the detector sufficiently that our diffraction pattern remained oversampled. Fig. If shows the extreme field of view that we obtained. Though the center of the beam only traversed $231 \mu \mathrm{m}^{2}$, a full $1375 \mu \mathrm{m}^{2}-6 \mathrm{x}$ larger than the scanned area-reconstructs well and features within the beam's $1 / \mathrm{e}^{4}$ extent, totaling $>9800 \mu \mathrm{m}^{2}(10 \mathrm{x}$ larger than the scanned area), accurately reconstruct. Lineouts taken through the structures support a resolution of $77.5 \mathrm{~nm}$ (vertical) x $265 \mathrm{~nm}$ (horizontal).

To reconstruct this object, we up-sample our diffraction patterns by $8 \mathrm{x}$ in the horizontal direction before applying conical diffraction curvature correction to ensure that they remain oversampled [5]. We then use ePIE [4] in combination with a technique we term Amplitude Constraint Relaxation (ACR), in which we impose the ptychographic amplitude constraint only on pixels whose guessed amplitude falls outside of some "acceptable bracket" of the recorded data. This bracket is minimally 1 detector count (to account for the camera's bit depth) and typically several standard deviations of the noise floor.

We have demonstrated a promising modality of high-resolution, wide field of view, tabletop imaging of thick, extended samples using $<13 \mathrm{~nm}$ light. We believe this technique is exciting for a variety of surface science, quantitative imaging, and semiconductor inspection applications.

\section{References:}

[1] J. Miao et al, Science, 348 (2015), pp. 530-5.

[2] B. Zhang et al, Ultramicroscopy, 158 (2015) pp. 98-104.

[3] J. R. Fienup, Appl. Opt. 52 (2013) pp. 45-56.

[4] A. M. Maiden and J. M. Rodenburg, Ultramicroscopy 109 (2009), pp. 1256-62.

[5] D. F. Gardner et al, Opt. Express, 20 (2012), pp. 19050-19059.

[6] The authors acknowledge major support from the following grants: DARPA PULSE W31P4Q-13-10015, a Gordon and Betty Moore Foundation EPiQS Initiative through Grant GBMF4538, an NSF Science and Technology Center DMR- 1548924, and DOE STTR Grant No. DE-SC0006514. 